\title{
UPGRADE STUDY OF INR PROTON LINAC FOR PRODUCTION OF 3 MW BEAM
}

\author{
$\underline{\text { L. V. Kravchuk }}^{\#}$ and P. N. Ostroumov \\ Institute for Nuclear Research RAS, 117312, Moscow
}

\begin{abstract}
There are many proposals for the construction of proton linacs with beam power up to $200 \mathrm{MW}$ for various applications (energy production, nuclear waste transmutation, etc...). In Russia there is a discussion of the necessity for the construction of the proton linac, which must exceed the beam power by one order of magnitude with respect to the existing linacs. Such linac must produce $1 \mathrm{GeV}$ energy beam with a $3 \mathrm{~mA}$ average current. The existing facility in the Institute for Nuclear Research allows an upgrade of the linac for the acceleration of higher intensity beam. The facility consists of a high intensity proton linac and an experimental area. The latter has been constructed for the transportation of several high intensity beams inside a 200 $\mathrm{mm}$ aperture beamline. Therefore it can be safely used for the transportation of very high power beam to target. For the production of a $3 \mathrm{~mA}$ beam it is proposed to extend the duty factor of the linac up to $6 \%$. The accelerating structure above $200 \mathrm{MeV}$ must be replaced by a superconducting structure operating at $594.6 \mathrm{MHz}$. The surface field of $17 \mathrm{MV} / \mathrm{m}$ in the superconducting structure provides acceleration of protons up to $1 \mathrm{GeV}$ over the length of the existing linac.
\end{abstract}

\section{INTRODUCTION}

The nuclear waste transmutation concept based on the high power linear accelerator system is firmly supported by the Russian government and is considered as a high priority task for the next decade. The full-power linac requires about $100 \mathrm{~mA} \mathrm{CW}$ current at $1 \mathrm{GeV}$ beam energy. Successful operation of superconducting RF (SRF) structures for the last two decades motivates the use of SRF cavities for such linac in the energy range above $200 \mathrm{MeV}$. There are two linacs in the world operating with high intensity beams. The most powerful linac at LANL can produce $0.8 \mathrm{MW}$ beam. The linear accelerator of the Moscow Meson Factory (MMF) produces $100 \mu \mathrm{A}$ beam at $500 \mathrm{MeV}$ (the specified energy is $600 \mathrm{MeV}$ ). The next generation of high-intensity linacs is designed for much higher beam power. From the practical point of view the construction of the demonstration linac with intermediate beam power of $\sim 5$ MW is necessary. This paper deals with the study of the MMF linac upgrade in order to produce $3 \mathrm{MW}$ beam. Its purpose is to confirm beam performance parameters, demonstrate an operation of SRF cavities and identify component failure modes.

The main task for the $3 \mathrm{MW}$ linac is the development of transmutation technologies. After this problem will be successfully solved, the linac can be switched to the acceleration of $\mathrm{H}^{-}$beam and can be transformed to a powerful spallation neutron source based on a compressor ring. For this purpose the linac will require the modification of its front end for the installation of an adequate chopper. However the laser stripping technology of the $\mathrm{H}^{-}$beam is under good progress, therefore even chopper will not be required in order to operate as a neutron source.

\section{SHORT OVERVIEW OF THE MMF LINAC}

The MMF linac consists of three main parts: injector system, DTL up to $100 \mathrm{MeV}$ and DAW cavities up to 600 $\mathrm{MeV}$ [1]. The general layout is shown in fig.1. The injector system includes $\mathrm{H}^{+}$and $\mathrm{H}^{-}$ion sources with high voltage transformers, two transport channels with

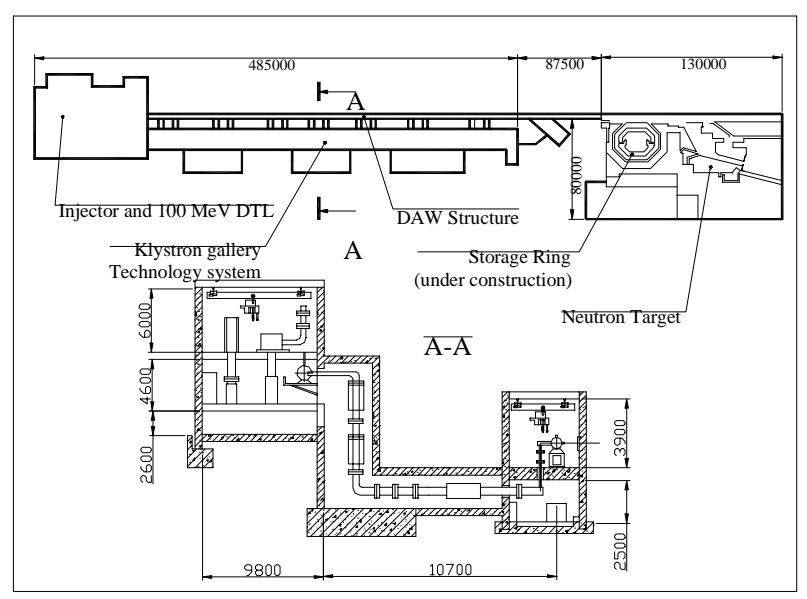

Figure 1: General layout of the MMF accelerator facility. The buildings of the auxiliary systems are not shown.

funneling magnet at $400 \mathrm{keV}$ and booster RFQ in the energy range from $400 \mathrm{keV}$ to $750 \mathrm{keV}$. The DTL operates at $198.2 \mathrm{MHz}$ and accelerates the beam from 0.75 to $100 \mathrm{MeV}$. The DAW structure operates at 991 $\mathrm{MHz}$ and accelerates the beam up to $600 \mathrm{MeV}$.

\footnotetext{
\# Email: kravchuk@al20.inr.troitsk.ru
} 
Originally the high voltage pulsed transformer has been used for acceleration up to $750 \mathrm{keV}$. The beam pulse length has been limited to $\sim 80 \mu$ s due to the transformer saturation. In order to increase the duty cycle of the beam from $0.8 \%$ to $1.5 \%$ as well as to allow the simultaneous acceleration of two beams, $\mathrm{H}^{+}$and $\mathrm{H}^{-}$, the booster RFQ in the energy range from $400 \mathrm{keV}$ to $750 \mathrm{keV}$ has been installed and commissioned recently.

The MMF linac operates typically with 70-100 $\mu \mathrm{A}$ average current for the proton beam at repetition rate of $50 \mathrm{~Hz}$. However, the linac can operate at $100 \mathrm{~Hz}$ with a corresponding increase of the average current, if necessary. A further increase of the average beam current can be achieved by the acceleration of the beam with peak current of $20 \mathrm{~mA}$. The latter is restricted by the DTL rf power system. We consider the MMF linac as the best candidate for the cheapest realization of a $3 \mathrm{MW}$ demonstration linac for the following reasons:

- No capital investment is required for the construction of new buildings for the linac and auxiliary systems.

- The DTL part will remain the old one. The water cooling system is able to operate with an $8 \%$ duty factor of the DTL and 198.2 MHz rf system.

- The experimental area of the MMF has been designed for the simultaneous transport of seven separate beams in the same aperture. It will allow a safe transport of $3 \mathrm{MW}$ beam to the target position.

- The target can be placed inside of the existing neutron source. A light modification of the shield will be required.

\section{THE PROPOSED MODIFICATIONS}

The general layout of the upgrade linac is shown in Fig. 2. The main beam parameters of the $3 \mathrm{MW}$ linac listed in Table 1 are achieved with the following modifications of the MMF linac:
- An RFQ with the proton ion source installed directly upstream of the $100 \mathrm{MeV}$ DTL;

- The main part of the linac from $100 \mathrm{MeV}$ to $1 \mathrm{GeV}$ contains room temperature Coupled Cavity Linac (CCL) up to $205 \mathrm{MeV}$ and Superconducting Linac (SCL) for acceleration to the final energy. The operating frequency both for CCL and SCL is 594.6 MHz. The new klystrons, high voltage transformers and waveguides can be located in the existing buildings (see Fig. 1).

Table 1: Basic parameters of the $3 \mathrm{MW}$ linac

\begin{tabular}{|l|c|}
\hline Beam energy & $1 \mathrm{GeV}$ \\
\hline Average current & $3 \mathrm{~mA}$ \\
\hline Pulsed current & $50 \mathrm{~mA}$ \\
\hline Beam pulse duration & $600 \mu \mathrm{s}$ \\
\hline Repetition rate & $100 \mathrm{~Hz}$ \\
\hline Number of RF stations at $198.2 \mathrm{MHz}$ & 5 \\
\hline Number of RF stations at $594.6 \mathrm{MHz}$ & 49 \\
\hline Number of cryostats with $\beta_{\mathrm{G}}=0.6$ & 28 \\
\hline Number of cryostats with $\beta_{\mathrm{G}}=0.8$ & 60 \\
\hline Total length of the linac & $464 \mathrm{~m}$ \\
\hline Number of SRF cells & 880 \\
\hline
\end{tabular}

The existing 198.2 MHz triodes GI-54 allow the generation of $3.5 \mathrm{MW}$ pulsed power with a duty factor of $8 \%$. However these triodes are no longer available. The DTL tanks are supplied by two rf windows, therefore the power can be generated in two rf stations and added in the cavity. For this purpose the commercially available triodes from "Svetlana" can be used.

The most important modifications are on the high energy part of the linac above $100 \mathrm{MeV}$. Due to the lack of proven technology of the superconducting rf (SRF) structures in the energy range between 100 to $200 \mathrm{MeV}$ we consider the use one of two types of accelerating

\section{Superconducting linac, $\mathrm{W}=1 \mathrm{GeV},<\mathrm{I}>=3 \mathrm{~mA}$}

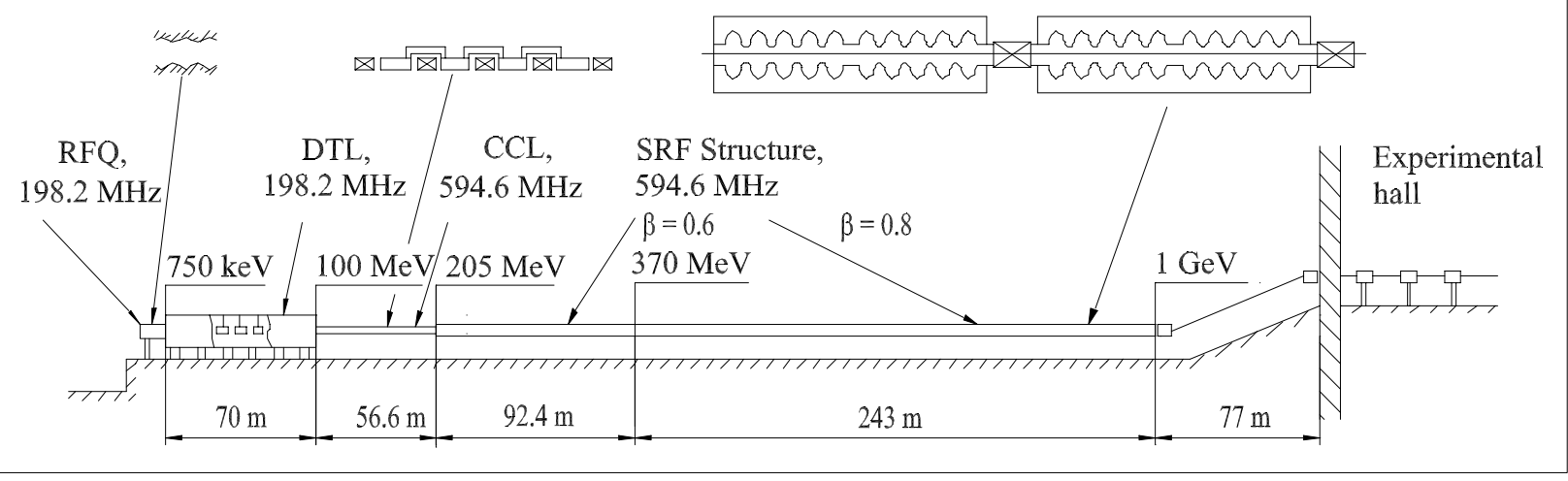

Figure 2: General layout of the $3 \mathrm{MW}$ linac to be located in the MMF. 
structures: the Disk and Washer Structure (DAW) [2] and the Cut Disk Structure CDS [3]. The CDS structure is very similar to the DAW structure as it concerned the electrodynamics parameters and vacuum conductivity. However the CDS is much compact and the cell diameter is only $\sim 360 \mathrm{~mm}$ at $594.6 \mathrm{MHz}$. The CDS is simpler for the manufacture but requires more R\&D for the operation with a high duty factor. The final decision for the CCL structure can be made later.

For the feeding both the CCL and SCL a $1.8 \mathrm{MW}$ klystron operating at $594.6 \mathrm{MHz}$ with anode modulation is considered.

The 198.2 MHz RFQ and transition energy of $750 \mathrm{keV}$ is the reasonable choice for the matching of a $50 \mathrm{~mA}$ peak current beam. The first 44 periods of the DTL are made with a $15 \mathrm{~mm}$ aperture diameter which restricts the normalized acceptance of the first DTL to $8 \pi \cdot \mathrm{mm} \cdot \mathrm{mrad}$. This allows scrapping of the beam halo at low energies and preserving total emittance low along the whole linac. The MMF linac originally has been designed for the acceleration of a $50 \mathrm{~mA}$ beam. The length of the focusing period of $2 \beta \lambda_{0}\left(\lambda_{0}\right.$ is the rf wavelength of the fundamental frequency) and zero current phase advance $\sigma_{0 \mathrm{t}}=45^{\circ}$ are quite reasonable for the focusing of a $50 \mathrm{~mA}$ beam. At the beginning of the SCL the focusing period length is $\sim 4 \beta \lambda_{0}$. Therefore the CCL will be designed for adiabatic matching of the transverse focusing properties. It will require the variation along the CCL the phase advance over the focusing period and focusing length counted in $\beta \lambda_{0}$. Both the DAW and the CDS are the best options for the high current acceleration due to the high coupling which is more than $30 \%$. For the $6 \%$ duty factor the accelerating gradient $\sim 3.5 \mathrm{MV} / \mathrm{m}$ of the CDS is restricted by the heat removal from the copper. The CCL will serve as an adiabatic matching in the longitudinal phase space. Therefore the use of the klystron power in the CCL cavities will be somewhat not optimal.

The SCL consists of two types of the SRF cavities with fixed geometrical beta: $\beta_{\mathrm{G}}=0.6$ and $\beta_{\mathrm{G}}=0.8$. The 5-cell cavities are best for the energy range from $200 \mathrm{MeV}$ to 1 $\mathrm{GeV}$. The sketch of the SRF cavities is shown in Fig. 3. Table 2 shows the basic parameters of the SCL.

Table 2: Basic parameters of the SCL

\begin{tabular}{|l|c|c|}
\hline Geometrical $\beta_{\mathrm{G}}$ & 0.6 & 0.8 \\
\hline Operating frequency, MHz & 594.6 & 594.6 \\
\hline Number of cells in SRF cavity & 5 & 5 \\
\hline Number of cavities in cryostat & 2 & 2 \\
\hline $\begin{array}{l}\text { Maximum electric field on the } \\
\text { surface, } \mathrm{MV} / \mathrm{m}\end{array}$ & 16.8 & 16.8 \\
\hline $\begin{array}{l}\text { Accelerating field (over the } \\
\left.\text { length of } 5 \beta_{\mathrm{G}} \lambda / 2 \mathrm{MV} / \mathrm{m}\right)\end{array}$ & 6.0 & 7.3 \\
\hline $\mathrm{TTF}$ & $\geq 0.7$ & $\geq 0.76$ \\
\hline Synchronous phase & $-32^{\circ}$ & $-32^{\circ}$ \\
\hline
\end{tabular}

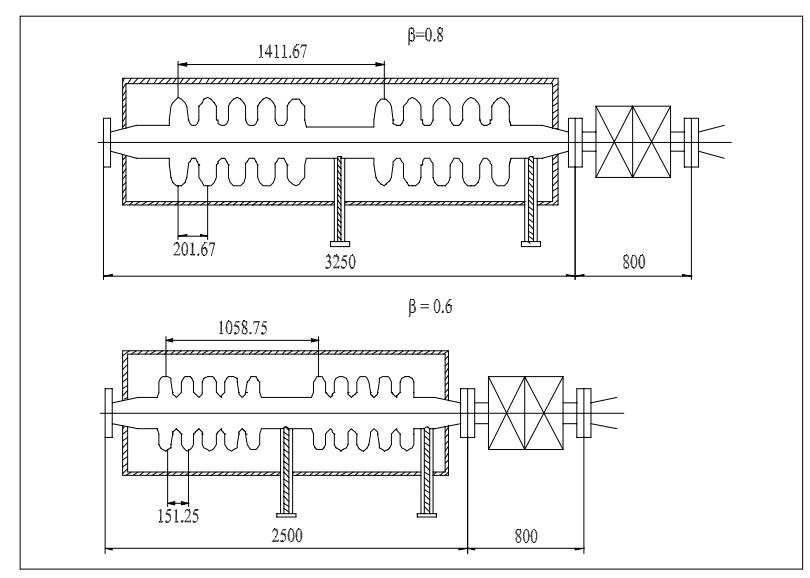

Figure 3: Two types of cryostats for a $1 \mathrm{GeV}$ linac.

The accelerating gradient along the whole linac averaged over the focusing period is shown in Fig. 4.

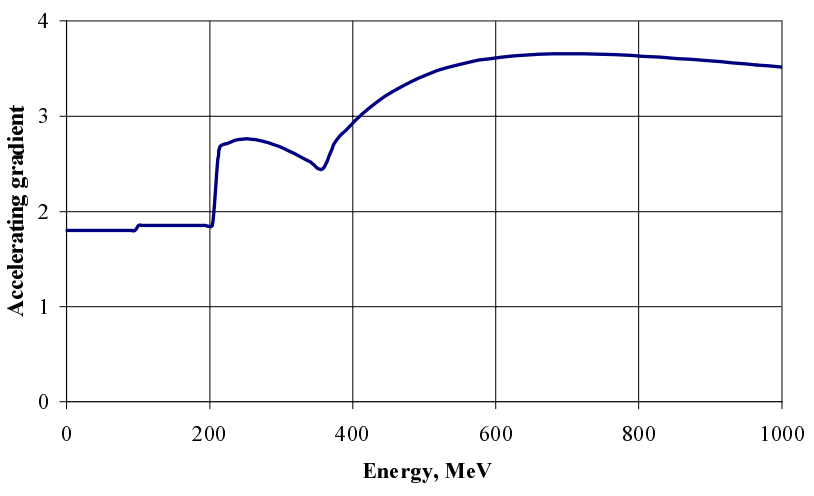

Figure 4: Average accelerating gradient along the linac.

\section{ACKNOWLEDGEMENT}

The authors thank their colleagues V.N. Leont'ev, A.I. Kvasha and V.V. Paramonov for helpful discussions.

\section{REFERENCES}

[1] S. K. Esin, L. V. Kravchuk, P. N. Ostroumov et. al. Commissioning/ Operation of the Moscow Meson Factory Linac. Proc. of the 1994 Linac Conf. August 21-26, Tsukuba, Japan, pp.31-35.

[2] V. G. Andreev et al. Study of High-Energy Proton Linac Structures. Proc. of the 1972 proton Linear Accel. Conf., p.114, LA5115, 1972.

[3] L. V. Kravchuk, V. V. Paramonov, V. A. Puntus. The Cold Model of the Cut Disk Structure. Proc. of the 1998 Linac Conf. To be published. 